\title{
Antenna Advancement Techniques and Integration of RFID Electronics on Organic Substrates for UHF RFID Applications in Automotive Sensing and Vehicle Security
}

\author{
Li Yang, Amin Rida, Jiexin Li, and Manos M. Tentzeris \\ Georgia Electronic Design Center, School of Electrical and Computer Engineering, \\ Georgia Institute of Technology, Atlanta, GA 30332-0250, USA \\ E-mail: liyang@ece.gatech.edu
}

\begin{abstract}
In this paper, design requirements and advanced approaches for improved performance UHF radio frequency identification (RFID) tags are presented. Organic substrates are examined as highly potential candidates for RFID fabrications. An inkjet-printed tag on paper substrate is realized for ultra-lowcost mass production. RFID with sensor integration is also demonstrated for automotive sensing and vehicle security applications. Measurements are performed for RFID tags embedded in tires. These results show that proper designed RFID can serve for the purpose of tire pressure/temperature monitoring.

Index Terms - RFID, sensor, organic substrate, inkjetprinting, paper substrate, automotive sensing, vehicle security.
\end{abstract}

\section{INTRODUCTION}

The demand for RFID implementations has recently increased tremendously due to the requirements of automatic identification in various areas, such as item-level tracking, access control, electronic toll collection, automotive sensing and vehicle security. Compared with the lower frequency tags (LF and HF bands) already suffering from limited read range (1-2 feet), RFID tags in UHF band see the widest use due to their higher read range (over 10 feet) and higher data transfer rate (currently $640 \mathrm{kbps}$ for Gen 2 RFID standard).

Three major challenges exist in today's RFID technologies. One is the design of small-size tag antennas with very high efficiency and effective impedance matching for IC chips with typically high capacitive reactance [1]. This antenna requirement is essential to optimize the RFID system powerperformance, especially for passive or semi-active configurations, where the only energy source is the incoming reader energy. Another major challenge is the existence of various different UHF frequency bands ranging from 866 to $954 \mathrm{MHz}$ for Europe (866-868 MHz), Asia (864-954 MHz) and US (902-928 MHz). The universal operation of the RFID necessitates the use of wideband antennas covering all three bands. The third obstacle is the realization of ultra-low-cost RFID tags, with a cost requirement for individual tags below one cent [2].

Antenna advancement design techniques, including adaptive impedance matching, universal operation design, directivity enhancement and dual polarization configuration for rugged environments, will be discussed in this paper. Embodiments, such as the North America/Europe bands RFID tags shown in Fig. 1, will be illustrated. In addition, integration of IC, sensors and a power supply in/on organic substrates that will benefit novel and existing automotive sensing and vehicle security applications will be demonstrated. More details will be elaborated at the conference.

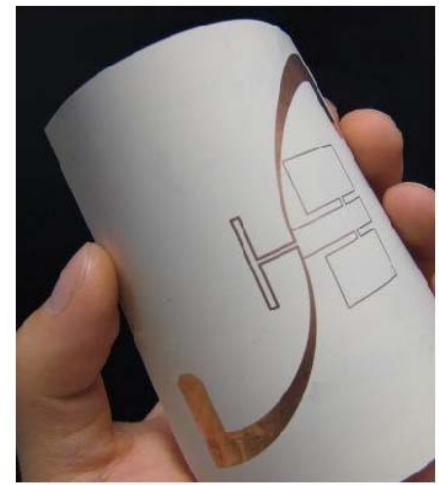

(a)

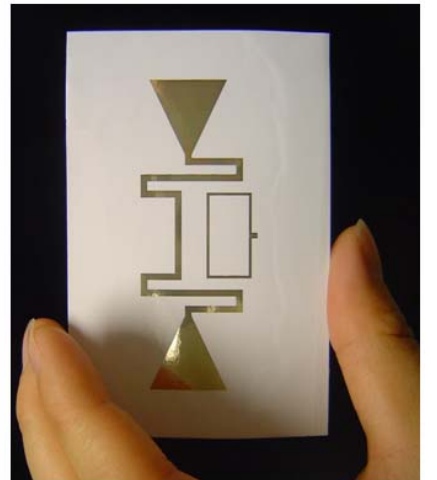

(b)
Fig. 1. Two wide band RFID tag antenna designs operating on both North America and Europe RFID bands. Adaptive impedance matching techniques are used: (a) serial stub feed structure (b) inductively coupled feed structure.

\section{RFID/SENSORS ON ORGANIC SUBSTRATES: PAPER}

Paper is considered as one of the best organic substrates for RFID applications for several reasons. Paper is not just environmentally friendly but can also undergo large reel to reel processing and is one of the cheapest materials known. Paper can also be made hydrophobic by applying the right layer on either or both sides. Dielectric constant of paper is close to air's (only 5-6\% power reflection) meaning electromagnetic power can penetrate easily even if the RFID is embedded in the substrate. Not to mention its flexibility to be embedded in hush environments like automobile tires in which paper can host nano-scale additives to contain fire retardant textiles and sustain temperature up to $200^{\circ} \mathrm{C}$. Furthermore, the low surface profile of paper with the appropriate coating makes paper compatible with fast printing processes, such as conductive paste inkjet printing instead of 
metal etching techniques, which will save a tremendous amount of time in the fabrication process. This also makes the RFID system much more secure since all fabrication processes can be done "on site". This means that the customer may print the design of the RFID module on paper substrates and with an appropriate IC integration process such as surface mount, assembly of a passive RFID tag can be accomplished. This eliminates any chance of counterfeiting ICs and avoiding any modifications (data or memory storage) to be made to ICs before arriving to the customer.

Active tags such as sensors and batteries can also be printed on paper in a multilayer fashion. Paper substrate can excellently handle high temperature treatment during the assembly process and the reliability/life time is very high compared to other substrates such as plastic. Paper can also be characterized easily for electrical properties using resonator structures [3].

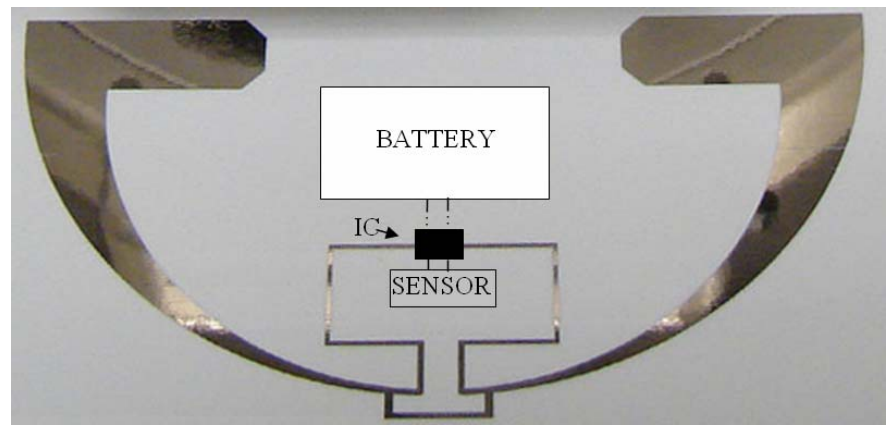

Fig. 2. Suggested outline of an inkjet-printed U-shaped antenna integrated with IC, sensor and embedded battery.

A suggested module of a printed UHF RFID active tag with sensor integration is shown in Fig. 2. The overall dimensions of the RFID tag shown below is: $8.2 \mathrm{~cm} \times 4.5 \mathrm{~cm}$.

\section{RFID/SENSORS FOR AUTOMOTIVE SENSING EMBEDDED IN TIRES}

Since the US Congress enacted the Transportation Recall Enhancement, Accountability, and Documentation Act ( TREAD Act ), motor vehicles have been required to equip with a system that warns the operator when tires are significantly underinflated. But general ABS system can only detect tire pressure drop larger than $25 \%$, and it can not identify the individual tire with a problem. Therefore, RFID tags integrated with sensors are highly desired for such application.

The tags are embedded in tires to monitor the pressure/temperature variation, and transmit information out wirelessly. These tags could improve consumer safety by providing information quickly and accurately. Especially, with the unique tag ID, when a recall occurs, the tires in question can be more easily targeted to an individual vehicle.

A testbed has been set upped for performance measurement of tires with RFID tags embedded. A RFID reader is located outside the tire and kept sending arrogating signals. The transmitted power is 4W EIRP in accordance with North America UHF Gen2 Regulation. After receiving the arrogating signals, the tag embedded in the tire will feed back its ID information. Effective communication length is recorded at different tire rotation angles. Part of the measured results is illustrated in Fig. 3.
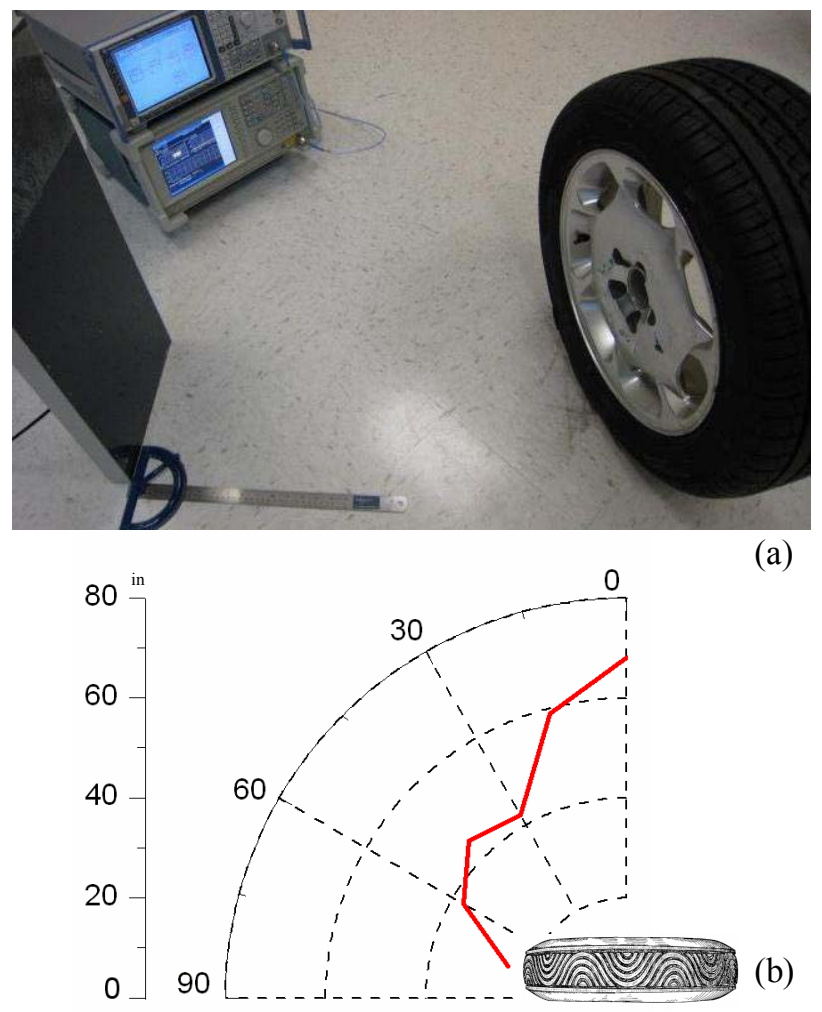

(a)

Fig. 3. (a) Measurement setup for tire with RFID tag embedded. (b) Effective length measured results.

During the vehicle assembly process, Vehicle Identification Number (VIN) can also be added to the RFID tag memory. The information then can be downloaded into a database, matching every tire to the vehicle on which it is installed - in effect creating a vehicle "fingerprint". The history of the vehicle then can be tracked. Theft cars can be easily located at toll stations equipped with tire RFID readers, and vehicle security will be heavily enhanced.

\section{REFERENCES}

[1] L. Yang, S. Basat, and M. M. Tentzeris, "Design and Development of Novel Inductively Couple RFID Antennas", Procs. of the 2006 IEEE-APS Symposium, pp.1035-1038, Albuquerque, NM, July 2006.

[2] V. Subramanian, J. M. Frechet, and etc., "Progress Toward Development of All-printed RFID Tags: Materials, Processes, and Devices," Proceedings of the IEEE, vol. 93, pp. $1330-$ 1338, No. 7, July 2005.

[3] D. Thompson, G. Ponchak, M. M. Tentzeris, J. Papapolymerou, "Characterization of LCP material and transmission lines on LCP substrates from 30 to $110 \mathrm{GHz}, "$ IEEE Trans. Microwave Theory \& Tech., vol. 52, no. 4, pp. 1343-1352, April 2004. 